\title{
Modellprojekte der Integrativen Onkologie
}

\author{
Gustav Dobos $^{\mathrm{a}} \quad$ Martin Wilhelm $^{\mathrm{b}}$ \\ ${ }^{a}$ Klinik Innere Medizin V, Naturheilkunde und Integrative Medizin, Kliniken Essen-Mitte, \\ ${ }^{\mathrm{b}}$ Medizinische Klinik 5, Schwerpunkt Onkologie/Hämatologie, Klinikum Nürnberg, Deutschland
}

\section{Das Kompetenznetz «Komplementärmedizin in der Onkologie - KOKON» \\ Verantwortlicher Autor: Martin Wilhelm, Nürnberg}

\section{Hintergrund}

Das Therapieangebot in der Komplementärmedizin ist nahezu unüberschaubar und reicht von Nahrungsergänzungsmitteln über diätetische Empfehlungen, Phytotherapien, Gewebeextrakte und manuelle Therapien bis zu hin zu psychologischen und psychosozialen Interventionen und komplexen Behandlungsverfahren fremder Kulturkreise.

Aktuelle Untersuchungen zeigen, dass 40\% der Krebspatienten Behandlungen aus diesen Bereichen in Anspruch nehmen, häufig ohne dass dies während der onkologischen Behandlung thematisiert wird [1].

Für einige dieser Verfahren werden auf der Basis randomisierter und kontrollierter Studien bereits eindeutige Empfehlungen für die klinisch-onkologische Praxis ausgesprochen etwa für die Elektroakupunktur zur Behandlung der Chemotherapie-induzierten Übelkeit [2], wohingegen die Evidenz zur Wirksamkeit und Sicherheit anderer Therapieverfahren teilweise nur aus Fallberichten stammt und als wenig verlässlich anzusehen ist.

Für Betroffene scheitert die Suche nach zuverlässigen Daten bereits an der Fülle des Materials; so gibt es etwa zur Misteltherapie rund 160000 deutschsprachige Treffer in Internet-Suchmaschinen - eine Überforderung für jeden, der auf diese Weise verlässliche Informationen sucht. Hinzu kommt, dass sich Fragen zur Komplementärmedizin nicht allein durch Verweise auf die Ergebnisse kontrollierter Studien (so es sie gibt) beantworten lassen.

So zeigen die langjährigen Erfahrungen aus der ärztlichen Beratungstätigkeit zur Komplementärmedizin am Klinikum Nürnberg, dass die Frage nach einer konkreten Therapiemaßnahme - z.B. zur Misteltherapie - oft nur der Aufhänger ist für weitergehende Gespräche, in denen es um andere Themen wie z.B. die Verträglichkeit der verordneten Krebsmedi- kamente geht oder auch um das Bedürfnis, durch Anwendung zusätzlicher Therapien etwas für sich selbst zu tun (Handlungsdrang), sowie um verdeckte Sorgen und Ängste.

Auf welche Anforderungen die Kommunikation über Komplementärmedizin trifft, kann durch eine Untersuchung von Beadle et al. [3] verdeutlicht werden: Sie weist darauf hin, dass Patienten, die komplementärmedizinische Verfahren nutzen, ein hohes Bedürfnis nach Kontrolle und Mitbestimmung bei Therapieentscheidungen haben und dass diese noch von der Chance auf Heilung ausgehen, auch wenn die Onkologen dies anders kommuniziert haben.

Beratung und Entscheidungen zu komplementärmedizinischen Verfahren setzen also nicht nur ein umfangreiches Wissen in der Onkologie und der Komplementärmedizin voraus, sondern bedeuten auch eine vielschichtige kommunikative Auseinandersetzung zwischen Therapeuten und Betroffenen, die in einem Spannungsfeld liegt zwischen Patientenautonomie und dem ärztlichen Bemühen, den Patienten zu unterstützen und vor Schaden zu schützen [4].

\section{Kompetenznetz Komplementärmedizin in der Onkologie}

Um die Qualität von Forschung und Versorgung im Bereich der Komplementärmedizin in der Onkologie zu verbessern, fördert die Deutsche Krebshilfe den Aufbau des deutschlandweiten, interdisziplinären Kompetenznetzes «Komplementärmedizin in der Onkologie - KOKON». An dem Kompetenznetz sind Institutionen und Fachleute der Universitäten Berlin, Frankfurt, Greifswald und Hamburg sowie der Universitätskliniken Freiburg, Hamburg-Eppendorf und Rostock beteiligt. Das Netzwerk wird im Sinne der Versorgungsforschung eine Analyse des Bedarfes an Information seitens onkologischer Patienten, der behandelnden Ärzte sowie des Pflegepersonals vornehmen, Fortbildungskonzepte für Fachleute und Schulungsprogramme für Patienten entwickeln sowie ein ärztliches Fachberatungsangebot etablieren, eine Wissensdatenbank zu Interaktionen, Nebenwirkungen und zur Evidenz komplementärmedizinischer Behandlungsverfah-

\section{KARGER}

Fax +497614520714

Information@Karger.de

www.karger.com (c) 2012 S. Karger GmbH, Freiburg

0378-584X/12/3517-0021\$38.00/0 
ren aufbauen und eine zentrale Informationsplattform zum Thema Komplementärmedizin in der Onkologie entwickeln. Ein Methodenzentrum wird alle wissenschaftlichen Untersuchungen supervidieren und auf gleichbleibend höchste Qualität der Abläufe sowie des Datenmanagements in den Teilprojekten achten. Die enge Vernetzung und Kooperation innerhalb von KOKON wird durch eine zentrale Koordinationsstelle gewährleistet und durch eine interdisziplinäre Gruppe von Fachleuten als Advisory Board begleitet und beraten.

Alle zentralen Projektabläufe im Kompetenznetz Komplementärmedizin in der Onkologie sind so konzipiert, dass deren Ergebnisse unmittelbar in der täglichen Versorgung von Krebspatientinnen und -patienten genutzt und umgesetzt werden können. Wir hoffen, dabei wie bisher auf die Unterstützung zahlreicher Institutionen bauen zu können.

\section{Das «Essener Modell»}

\section{Verantwortlicher Autor: Gustav Dobos, Essen}

Das «Essener Modell» beinhaltet ein Konzept zur integrativonkologischen Therapie, das sich in einer ersten Phase an Patientinnen mit Brustkrebs wendet. Es nutzt neben der konventionell bewährten Medizin (der sogenannten Schulmedizin) Verfahren der Naturheilkunde und der Mind-BodyMedizin, aus denen individuelle begleitende Behandlungsstrategien entwickelt werden. Unseren Beitrag zur Integrativen Onkologie sehen wir in Anlehnung an eine Definition der Society for Integrative Oncology bzw. des Department for Integrative Medicine am Memorial Sloan-Kettering Cancer Center (MSKCC) in New York in der Nutzung von Arzneimitteln und Therapieverfahren der wissenschaftlich geprüften Naturheilkunde zur Minderung von Nebenwirkungen, zur Verbesserung der Lebensqualität und zur Prävention vor und nach Krebs. Es handelt sich dabei nicht um alternative, sondern um begleitende Maßnahmen zu konventionellen onkologischen Therapien (Abb. 1).

Standort sind die Kliniken Essen-Mitte, akademisches Lehrkrankenhaus der Universität Duisburg-Essen, als eine Kooperation der Kliniken für Senologie (Leiter: PD Dr. Sherko Kümmel) bzw. für Naturheilkunde und Integrative Medizin am Knappschafts-Krankenhaus der Kliniken EssenMitte. Letztere wurde 1999 als Modelleinrichtung des Landes Nordrhein-Westfalen eingerichtet mit dem Ziel, optimale Behandlungsansätze aus konventioneller Medizin und wissenschaftlich geprüfter Naturheilkunde zu kombinieren. Zur Klinik gehören zwei internistische Stationen mit insgesamt 54 Betten, eine integrativ-onkologische Tagesklinik und das ihr angeschlossene ambulante Institut für Naturheilkunde, Traditionelle Chinesische und Indische Medizin sowie die Integrative Gastroenterologie.

Den Anstoß für das Projekt «Integrative Onkologie» gab die «Naturheilkundliche Sprechstunde bei Brustkrebs», die

\section{Integrative Medizin}

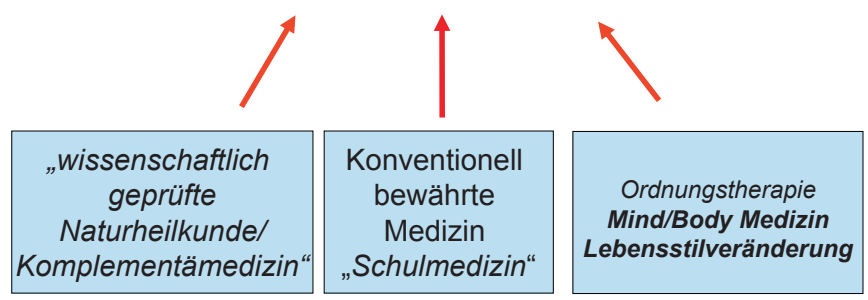

Abb. 1. Säulen der Integrativen Medizin.

von 2004 bis 2008 mit Unterstützung der Karl und Veronica Carstens-Stiftung an der Universitätsfrauenklinik Essen (Leiter: Prof. Dr. Rainer Kimmig) eingerichtet wurde und von Mitarbeitern der Modelleinrichtung der Kliniken Essen-Mitte durchgeführt wurde. In einem nächsten Schritt hat sich daraus 2010 das Projekt «Integrative Onkologie» an den Kliniken Essen-Mitte entwickelt, das sich zunächst auf die Senologie konzentrierte, seit Januar 2012 aber das gesamte gynäkologisch-onkologische Spektrum (Leiter: Prof. Andreas du Bois) abdeckt und ab Juni 2012 auch die Klinik für Internistische Onkologie/Hämatologie (Leiter: Prof. Hansjochen Wilke, Prof. Michael Stahl) einbeziehen wird.

Im Rahmen des Essener Modells werden Therapieansätze auf Wirksamkeit und Sicherheit evaluiert, des Weiteren wird das Konzept der Integrativen Onkologie auf praktische Umsetzbarkeit geprüft und die Forschung auf diesem Gebiet intensiviert. Zu den weiteren Aufgaben gehört die Mitarbeit an den Leitlinien der Arbeitsgemeinschaft Gynäkologische Onkologie (AGO) zur evidenzbasierten Einordnung komplementärer Verfahren wie Yoga, Meditation und Akupunktur sowie die Organisation von Fort- und Weiterbildungsveranstaltungen. In einem von der Robert Bosch Stiftung im Oktober 2011 geförderten Consensus-Workshop wurden unter Mitarbeit von 20 internationalen Experten die Grundlagen zur Erstellung eines Curriculums der Integrativen Onkologie sowie für Leitlinien zur Komplementärmedizin in der Onkologie erarbeitet. Mit der Einrichtung eines Leitlinienbüros an den Kliniken Essen-Mitte in Zusammenarbeit mit der Deutschen Gesellschaft für Naturheilkunde werden diese Vorhaben weiter vorangetrieben. Entsprechend wird vom 26. bis 28. Oktober 2012 die erste, auf den Empfehlungen des Expertenteams basierende Fort- und Weiterbildung «Integrative Onkologie» an den Kliniken Essen-Mitte angeboten.

Die «Integrative Medizin» in Essen wird als ein «2-Experten-Modell» praktiziert, in Anlehnung an das Kooperationsmodell des US-amerikanischen MSKCC. Das dortige Department for Integrative Medicine versorgt mit seinen 50 Mitarbeitern in Zusammenarbeit mit den weiteren onkologischen Kliniken über 17000 Patienten pro Jahr. Durch die enge Verflechtung zweier Abteilungen lassen sich integrative Therapieansätze nach unserer Erfahrung kompetenter und besser 


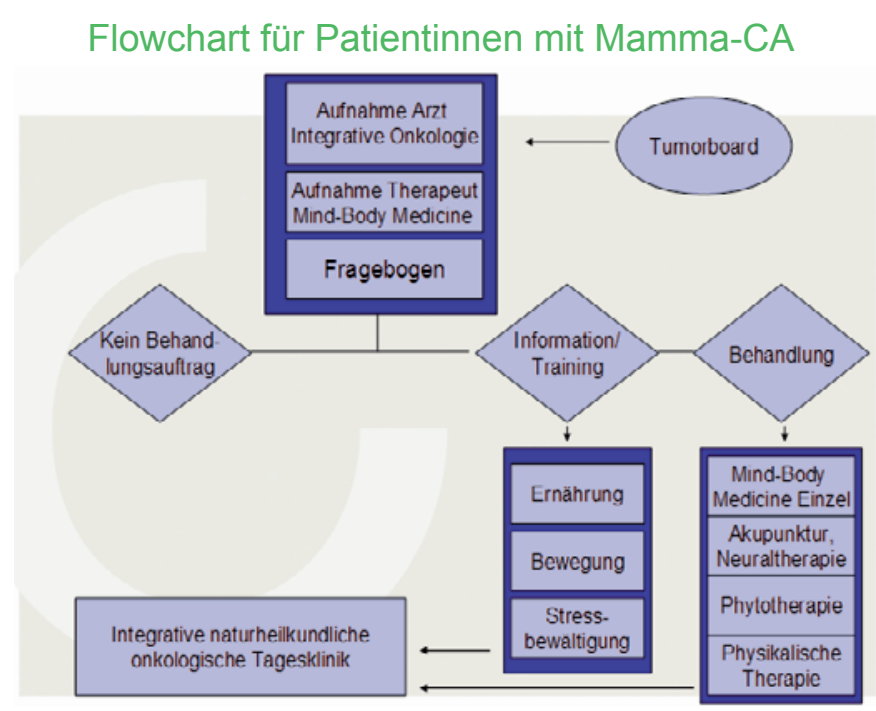

Abb. 2. Systematisches Vorgehen für Patientinnen mit Mammakarzinom.

in Kliniken etablieren, als wenn onkologische Abteilungen selbstständig lediglich einzelne komplementärmedizinische Zusatzmodule übernehmen.

Die «Integrative Onkologie» an den Kliniken Essen-Mitte nutzt seit 2010 ein datenbankgestütztes Expertensystem (SenoExpert), mit dem jederzeit auf aktuelle Studiendaten auch aus dem Bereich der Naturheilkunde zurückgegriffen werden kann. So lassen sich schon früh im Behandlungsverlauf auch komplementärmedizinische Ansätze in die Therapie evidenzbasiert integrieren. Dies erfolgt in enger Absprache mit den senologisch und gynäkologisch-onkologischen Abteilungen bzw. interdisziplinär in der Tumorkonferenz. Aus den Angeboten der Komplementärmedizin wird in Ergänzung zur onkologischen Behandlung nach einem systematischen Vorgehen (Abb. 2) die individuell passende, symptomorientierte komplementärmedizinische Therapie zusammengestellt. Etwa 75\% der Patientinnen nehmen das Angebot einer ergänzenden naturheilkundlichen Behandlung an, wobei meist bereits während des stationären Aufenthaltes eine ausführliche Beratung über das spezifische Nebenwirkungsmanagement konventioneller Therapien durch einen naturheilkundlich ausgerichteten Arzt stattfindet. Inhalte der etwa 1-stündigen Erstgespräche sind - je nach Patientenbedürfnis - die naturheilkundlichen Therapieansätze und/oder die Mind-Body-Medizin (etwa zur präoperativen Entspannung, Umgang mit Angst, Depression, Krankheitsbewältigung oder Erhaltung bzw. Wiedererlangung der körperlichen Fitness).

Das am häufigsten genutzte ambulante Therapieangebot ist die Akupunktur. Sie wird in der Regel während bzw. nach jeder Chemotherapie eingesetzt, sowie 2-mal während einer Strahlentherapie, 4-mal während einer endokrinen Therapie sowie nach Bedarf bei therapieassoziierten Schmerzen. Im Jahr 2011 fanden in unserer Abteilung mehr als 2000 Akupunktur-Sitzungen statt. In ähnlicher Häufigkeit wurden die Angebote der Mind-Body-Medizin genutzt. Der Einsatz von
Dests

Ziele: Integrative Onkologie

- Verbessertes Nebenwirkungsmanagement ...

- Verbesserte psychische und körperliche Fitness

- Verbesserung der LQ der Patienten (Reduktion von Depression, Stress-Erleben und Angst)

- Verbesserung der Compliance (seltenere TherapieAbbrüche)

- Primär- und Sekundärprophylaxe

- Verbesserung der Prognose?

KLINIKEN ESSEN-MITTE,
Department for Internal and Integrative Medicine

Abb. 3. Ziele der Integrativen Onkologie.

Akupunktur im Rahmen der Chemotherapie führt zu einer deutlichen Reduktion Chemotherapie-bedingter Nebenwirkungen. Gut belegt ist die Wirksamkeit von Akupunktur bei Gelenkschmerzen unter Therapie mit Aromatasehemmern [5]. Auch Hitzewallungen und andere Beschwerden unter antihormoneller Therapie werden durch Akupunktur deutlich und anhaltend gebessert [6].

Gemeinsam ist den Verfahren die Zielsetzung, Nebenwirkungen der konventionellen Therapien zu lindern und die Lebensqualität der Patientinnen zu verbessern (Abb. 3). Dass dies auch gelingt, zeigt die deutlich rückläufige Zahl an Therapieabbrüchen im Rahmen von Unverträglichkeiten, z.B. von antihormonellen Therapien. Ob sich durch integrative Ansätze auch ehrgeizige Ziele wie etwa eine Prognoseverbesserung erreichen lassen, müssen künftige Studien zeigen.

Innerhalb der Komplementärmedizin konzentriert sich die sogenannte Mind-Body-Medizin auf Verfahren, die bei der mentalen und psychischen Verarbeitung des Krankheitsgeschehens helfen (s. Beitrag Paul). Dabei geht es im Wesentlichen um Aspekte der körperlichen Bewegung, der Ernährung und des Umgangs mit Stress. Durch Kombination der Maßnahmen kann ein zusätzlicher Benefit erzielt werden, wie unter anderem in einer Studie von Pierce et al. [7] gezeigt werden konnte: Die prospektive Studie wies bei Patientinnen mit operiertem Brustkrebs nach, dass ein moderates Sportprogramm (3 Stunden pro Woche) in Kombination mit gesunder Ernährung (5 Portionen Obst oder Gemüse täglich) das 10-Jahres-Mortalitätsrisiko halbiert. Des Weiteren konnten für achtsamkeitsbasierte Therapien, wie der mindfulnessbased stress reduction (MBSR), für Yoga und für Qigong eine gute Evidenzlage gezeigt werden. Entsprechend wurden diese Therapien im Jahr 2011 in den AGO-Leitlinien-Katalog aufgenommen.

Naturheilkundliche bzw. komplementäre Therapieansätze sind besonders hilfreich, um Patienten psychisch zu 
entlasten und einer Depression vorzubeugen. Eine krankheitsbegleitende «Major Depression» kann nach einer Metaanalyse von Satin et al. [8] eine um bis zu 39\% erhöhte Mortalität zur Folge haben. Studien belegen die antidepressive Wirkung von Sport/Bewegung [9], von Achtsamkeitsmeditation [10], von Yoga [11], von Programmen zur Gruppenunterstützung [12] und von diätetischen Maßnahmen mit Anhebung des Anteils von Omega-3-Fettsäuren in der Ernährung [13]. Im Gegensatz dazu muss bei Einsatz von medikamentösen antidepressiven Therapien (sowohl allopathisch als auch phytotherapeutisch) in jedem Fall die Interaktion mit der jeweiligen Chemotherapie geprüft werden.

Naturheilkundliche Therapieansätze werden als Intensivprogramme von der Tagesklinik angeboten. Insbesondere die Mind-Body-Techniken und die damit angestrebte Lebensstiländerung lassen sich nicht von heute auf morgen erlernen bewährt hat sich das in Anlehnung an Konzepte des Mind/ Body Medical Institute der Harvard Medical School entwickelte ganztägige Gruppenprogramm über eine Dauer von 10 Wochen. Zu den Kursinhalten gehören die Information über die Krankheit, Lebensstilfaktoren, Achtsamkeitsschulung, Übungen zur Stressbewältigung und die Vermittlung von Selbsthilfestrategien.

Ob sich auch durch Einnahme von Phytotherapeutika die Verträglichkeit von Chemotherapien verbessern lässt, ist noch wenig untersucht. In einer prospektiven, placebokontrollierten Studie mit 65 an Brustkrebs erkrankten Patientinnen konnte durch Einnahme einer definierten Kräutermischung mit Chinesischen Kräutern (LCS101) die Hämatotoxizität einer Anthrazyklin-basierten Chemotherapie signifikant reduziert werden [14], wobei Einzelstudien dieser Art noch nicht ausreichen, um eine tragbare Aussage machen zu können. Von besonderer Bedeutung ist in diesem Zusammenhang der Blick auf mögliche Interaktionen zwischen Phytopharmaka und Chemotherapeutika. Sie betreffen z.B. das Cytochrom-P450-Enzymsystem, über das eine Vielzahl von Arzneimitteln metabolisiert wird. Induktoren des CYP-3A4Isoenzyms sind z.B. Johanniskraut, Ingwer und Knoblauch, gehemmt wird das Isoenzym etwa durch Baldrian, Ginseng und Gelbwurzel. Sogar grüner Tee kann die Wirkung einer Chemotherapie abschwächen, wie bei Patienten unter Therapie mit Bortezomib gezeigt worden ist [15]. Sehr hilfreich ist

\section{Begleitende Therapien}

\author{
Ernährung \\ Bewegung \\ Mind/Body Medizin (Yoga, Meditation Qigong, u.a.) \\ Mindfulness based stress reduction (MBSR) \\ Phytotherapie \\ Vitamine, Spurenelemente \\ Akupunktur \\ Mistel \\ Fußmassage
}

KLINIKEN ESSEN-MITTE,

Department for Internal and Integrative Medicine

Abb. 4. Komplementärmedizinische begleitende Therapieangebote für Patientinnen mit Brustkrebs.

die Internetplattform www.mskcc.org/AboutHerbs des MSKCC.

Im «Essener Modell» kommen grundsätzlich entsprechend den jeweiligen Therapiephasen die in Abbildung 4 gelisteten Therapien zum Einsatz.

Das «Essener Modell» ermöglicht die stationäre, tagesklinische und ambulante Versorgung von Krebspatienten nach dem Konzept der Integrativen Medizin und ist in dieser Form eine Novität in Deutschland. Durch den forschungsaktiven Hintergrund und die damit verbundene enge Koppelung der Einrichtung mit Forschung und Lehre ist eine zeitnahe Umsetzung neuer wissenschaftlicher Erkenntnisse im Bereich der Naturheilkunde gewährleistet. Die ab Oktober 2012 angebotenen zertifizierten Fort- und Weiterbildungsangebote im Bereich «Integrative Onkologie» erlauben die qualitätsgesicherte Umsetzung in deutschsprachigen onkologischen Einrichtungen (Informationen unter 0201/174 25512 oder per E-Mail: fortbildungen-nhk@kliniken-essen-mitte.de).

\section{Disclosure Statement}

Es bestehen keine Interessenkonflikte.

\section{Literatur}

1 Horneber M, Büschel G, Dennert G, Less DB, Ritter E, Zwahlen M: How many cancer patients use complementary and alternative medicine: a systematic review and meta-analysis. Integr Cancer Ther 2011; DOI: $10.1177 / 1534735411423920$.

$\checkmark 2$ Naeim A, et al.: Evidence-based recommendations for cancer nausea and vomiting. J Clin Oncol 2008; 26:3903-3910.
Beadle GF, et al.: Beliefs and practices of patients with advanced cancer: implications for communication. Br J Cancer 2004;91:254-257.

4 Horneber MA, Rostock M, Büschel G: Patientenberatung zu unkonventionellen Verfahren in der Krebstherapie; in Bartsch HH, Weis J (eds): Gemeinsame Entscheidung in der Krebstherapie. Basel, Karger, 2004, pp 91-94. $\checkmark 5$ Crew KD, et al.: Randomized, blinded, sham-controlled trial of acupuncture for the management of aromatase inhibitor-associated joint symptoms in women with early-stage breast cancer. J Clin Oncol 2010;28:1154-1160.

6 Walker EM, et al.: Acupuncture versus venlafaxine for the management of vasomotor symptoms in patients with hormone receptor-positive breast cancer: a randomized controlled trial. J Clin Oncol 2010;28:634-640. 
7 Pierce JP, et al.: Greater survival after breast cancer in physically active women with high vegetablefruit intake regardless of obesity. J Clin Oncol 2007; 25:2345-2351.

$\checkmark 8$ Satin JR, et al.: Depression as a predictor of disease progression and mortality in cancer patients: meta-analysis. Cancer 2009;115:5349-5361.

9 Mead GE, et al.: Exercise for depression. Cochrane Database Syst Rev 2009;(3):CD004366.

10 Chiesa A, Serretti A: Mindfulness-based stress reduction for stress management in healthy people: a review and meta-analysis. J Altern Complement Med 2009;15:593-600.
Pilkington K, et al.: Yoga for depression: the research evidence. J Affect Disord 2005;89:13-24.

12 Goodwin PJ, et al.: The effect of group psychosocial support on survival in metastatic breast cancer. N Engl J Med 2001;345:1719-1726.

13 Sarris J, et al.: Adjuvant use of nutritional and herbal medicines with antidepressants, mood stabilizers and benzodiazepines. J Psychiatr Res 2010; 44:32-41.
14 Yaal-Hahoshen N, et al.: A prospective, controlled study of the botanical compound mixture LCS101 for chemotherapy-induced hematological complications in breast cancer. Oncologist 2011;16:11971202.

5 Golden EB, et al.: Green tea polyphenols block the anticancer effects of bortezomib and other boronic acid-based proteasome inhibitors. Blood 2009;113: 5927-5937. 